\title{
Maternal-child overweight/obesity and undernutrition in Kenya: a geographic analysis
}

\author{
Lisa R Pawloski ${ }^{1, *}$, Kevin M Curtin ${ }^{2}$, Constance Gewa ${ }^{1}$ and David Attaway ${ }^{2}$ \\ 'Department of Nutrition and Food Studies (MSN 1F8), George Mason University, 4400 University Drive, Fairfax, \\ VA 22030, USA: ${ }^{2}$ Department of Geography and Geoinformation Science, George Mason University, Fairfax, VA, USA
}

Submitted 25 January 2011: Accepted 3 January 2012: First published online 14 March 2012

\begin{abstract}
Objective: The purpose of the study was to examine geographic relationships of nutritional status (BMI), including underweight, overweight and obesity, among Kenyan mothers and children.

Design: Spatial relationships were examined concerning BMI of the mothers and BMI-for-age percentiles of their children. These included spatial statistical measures of the clustering of segments of the population, in addition to inspection of co-location of significant clusters.

Setting: Rural and urban areas of Kenya, including the cities of Nairobi and Mombasa, and the Kisumu region.

Subjects: Mother-child pairs from Demographic and Health Survey data including 1541 observations in 2003 and 1592 observations in 2009. These mother-child pairs were organized into 399 locational clusters.

Results: There is extremely strong evidence that high BMI values exhibit strong spatial clustering. There were co-locations of overweight mothers and overweight children only in the Nairobi region, while both underweight mothers and children tended to cluster in rural areas. In Mombasa clusters of overweight mothers were associated with normal-weight children, while in the Kisumu region clusters of overweight children were associated with normal-weight mothers.

Conclusions: These findings show there is geographic variability as well as some defined patterns concerning the distribution of malnutrition among mothers and children in Kenya, and suggest the need for further geographic analyses concerning the potential factors which influence nutritional status in this population. In addition, the methods used in this research may be easily applied to other Demographic and Health Survey data in order to begin to understand the geographic determinants of health in low-income countries.
\end{abstract}

Keywords
Obesity
Clustering
Spatial analysis
Kenya
Geographic information systems

Geraphic informalion systems

Traditionally, public health challenges within developing countries have centred on issues related to infectious disease and undernutrition, which have been at the forefront of global health initiatives. This is no longer the case as, according to the WHO's latest review of obesity trends, approximately 1.5 billion adults were overweight and at least 500 million adults were obese in 2008. Additionally, it has been estimated that another 43 million children (under 5 years old) around the world were overweight in $2010^{(1)}$. Based on these figures, the WHO further projects that this nutrition transition (the phenomenon where a population shifts from heath patterns associated with undernutrition (e.g. infectious diseases) to health patterns associated with overnutrition (e.g. obesity and chronic diseases) ${ }^{(2)}$ ) will continue, and approximately $2 \cdot 3$ billion adults will be overweight and more than 700 million will be obese by $2015^{(3)}$.

While there have been recent investigations concerning the nutrition transition in South America and Asia ${ }^{(4)}$, few studies have examined the situation throughout subSaharan Africa. There are even fewer which have examined the obesity situation among children. Understanding the prevalence and causes of obesity among children is critical as obesity greatly increases the risk of developing a chronic disease during childhood, which may lead to significant complications lasting throughout adult life.

As Africa is faced with continuing challenges related to HIV/AIDS and other infectious diseases as well as undernutrition, there are few resources available to focus on the emerging nutrition transition. Most of the studies concerning obesity in Africa have been conducted in wealthier and more populated African nations in the Southern and Western regions of Africa, including South Africa $^{(5)}$, Botswana ${ }^{(6)}$, Cameroon ${ }^{(7)}$ and Benin ${ }^{(8)}$. However, even in one of the wealthiest countries of Africa, South Africa, stunting has been shown to coexist alongside obesity even within the same socio-geographic population ${ }^{(9)}$. 
Another phenomenon found in transitional countries is the coexistence of child undernutrition and maternal overweight within the same household. Bouzitou et al. have reported such a trend in the West African country of Benin $^{(10)}$. Garrett and Ruel have suggested that the coexistence of a stunted child and an overweight mother is associated with increasing economic development but not necessarily urbanization, and is a phenomenon more often seen in transitional countries ${ }^{(11)}$.

While the coexistence of overweight/obese mothers and stunted children is not an unusual pattern found in transitional countries, there are other more common patterns concerning mother and child nutritional status. In the USA recent research has pointed to maternal overweight being a risk factor for overweight children, particularly among Hispanic mothers ${ }^{(12)}$. Further, in Kenya, Gewa also noted that maternal overweight/obesity tends to be a significant predictor of childhood obesity ${ }^{(13)}$. Maternal overweight has been shown to increase the probability of obesity and complications in newborns, and to influence nutrition and health later in childhood, including an increased risk for diabetes and metabolic syndrome ${ }^{(14-16)}$. As obesity is more common in wealthier nations, so is the coexistence of maternal overweight/obesity with childhood overweight/obesity. However, this trend is important to note in transitional countries, which have recently experienced a growth in overweight/obesity.

Only a handful of studies concerning obesity have been conducted in East Africa. Gewa is one of the first authors to publish data concerning the rise of obesity in children in Kenya. Using data collected from the 2003 Demographic and Health Survey (DHS), her research found that approximately $22 \%$ of children aged 3 to 5 years were either overweight or obese. The factors associated with obesity were (i) having an overweight or obese mother, (ii) having a more educated mother and (iii) having a high birth weight. Factors that were negatively correlated with obesity were (i) being an older child and (ii) coming from a large household ${ }^{(13)}$.

Although Gewa has shown the relationship between physiological and sociodemographic factors influencing childhood obesity in Kenya, no studies have examined the socio-geographic factors related to childhood malnutrition. We define malnutrition as the condition of being undernourished or overnourished. While the use of geographic information systems to examine and encourage development in low-income countries is increasing (e.g. Holmes et $a l^{(17)}$ and Hill and Curtin ${ }^{(18)}$ ), there is no known such application in public health nutrition. Given that Kenya is an extremely diverse country geographically, it is critical to review such potential determinants. However, no study has yet examined the geographic distribution of maternal and child malnutrition. Thus using data from both the 2003 and the 2009 DHS, the present study examines the geographic distribution and patterns of nutritional indicators among Kenyan mothers and Kenyan children aged 3 to 5 years.

\section{Purpose and research questions}

The purpose of the study was to examine geographic relationships of nutritional status as measured by BMI for Kenyan mothers and by BMI-for-age percentiles (BAP) for their children.

In order to examine the geographic distribution of nutritional indicators among mothers and children in Kenya, the following three research questions were addressed:

1. Does a point pattern analysis of malnutrition in mothers and the same analysis of malnutrition in children show significant clustering of similar values?

2. What is the geographic distribution among malnourished mothers and children in Kenya?

3. Is there a geographic pattern regarding the coexistence of overweight/obese mothers and undernourished children?

\section{Participants and methods}

\section{Participants}

Data from both the 2003 and the 2009 DHS from Kenya were analysed. The DHS uses a multistage stratified cluster sampling methodology in which samples of households within clusters are selected. For these data a representative probability sample of almost 10000 households was selected for the surveys. Anthropometric measurements were taken from mother-child pairs. Each observation consists of a mother-child pair with the associated BMI and BAP values for the mother and child, respectively. The current analysis focuses on children aged 3 to 5 years, an age group where a majority (97\%) had been weaned off breast milk. A total of 1541 (2003) and 1592 (2009) mother-child pairs were included in the present analysis. The DHS protocol was approved by ORC Macro's Institutional Review Board.

Geographic coordinates of the participants were captured. The longitude and latitude coordinates were associated with the cluster location where participants were examined. Since the data were collected at cluster locations, there are between one and thirteen individuals with the same locational characteristics. The mother-child pairs were distributed among 399 clusters (in both data sets). Figure 1 provides a geographic reference showing the major cities (Kisumu, Nairobi and Mombasa) and the Northeast region, all of which figure prominently in the results presented herein. Kisumu is the third largest city in Kenya, behind Mombasa (second) and the capital Nairobi. The cities differ in their socio-cultural make-up in that Mombasa is inhabited largely by a Muslim Mijikenda/Swahili population, while most Kisumu residents are of the Luo ethnic group. Nairobi is much more diverse with multiple ethnic groups such as Kikuyu, Luo, Kalenjin, Kisii and others.

\section{Antbropometric indicators}

Heights and weights were taken by trained teams of enumerators. Weight was measured to the nearest $0.5 \mathrm{~kg}$ 


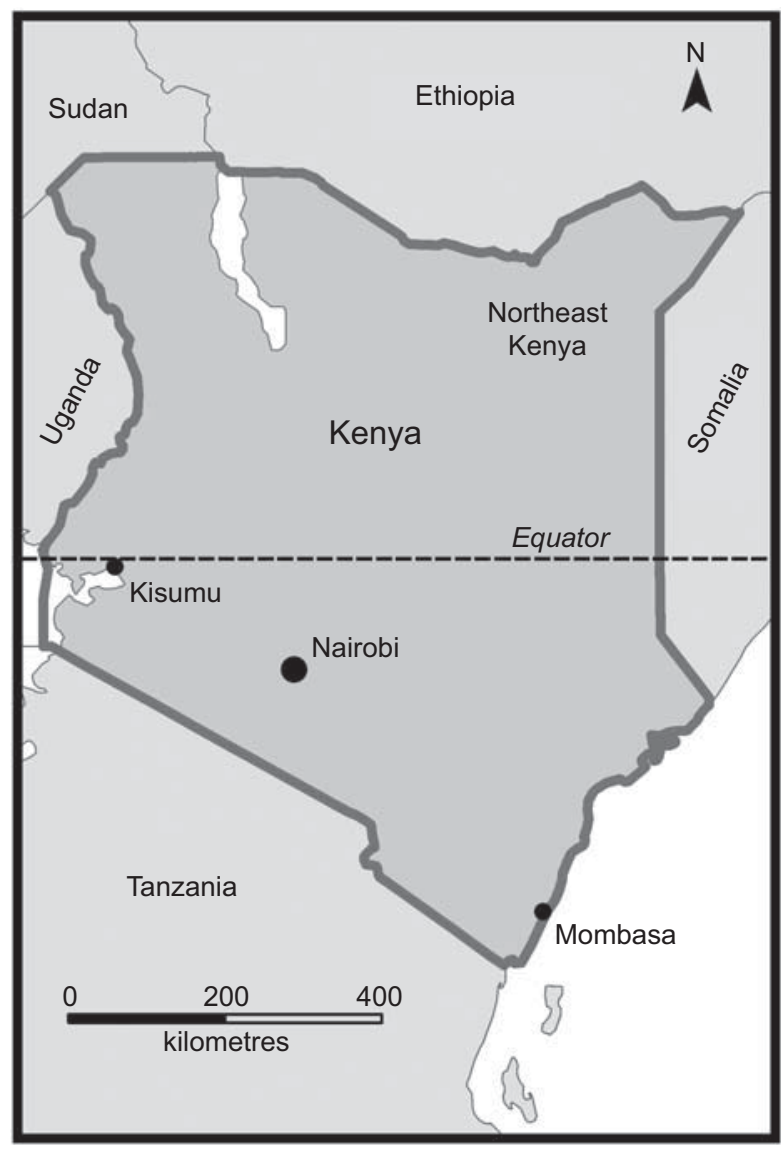

Fig. 1 Reference map of Kenya and its major cities

using a lightweight, bathroom-type scale with a digital screen designed under the authority of UNICEF. Height was measured to the nearest $0 \cdot 1 \mathrm{~cm}$ using a calibrated height board/flexible tape. The measuring boards were specially produced by Shorr Productions for use in survey settings. BAP values were generated using WHO 2006 growth reference standards which use the WHO Multicentre Growth Reference Study population. To classify overweight and obesity among children, overweight or obesity was defined as BAP $>85$ th percentile and underweight was defined as $\mathrm{BAP}<5$ th percentile ${ }^{(19)}$. Maternal BMI was computed by dividing weight in kilograms by the square of height in metres. BMI cut-offs were based on international recommended cut-offs ${ }^{(20)}$ : underweight was defined as $\mathrm{BMI}<18.5 \mathrm{~kg} / \mathrm{m}^{2}$, normal weight as BMI $=18 \cdot 5-24.9 \mathrm{~kg} / \mathrm{m}^{2}$ and overweight/obesity as $\mathrm{BMI} \geq 25 \cdot 0 \mathrm{~kg} / \mathrm{m}^{2}$.

\section{Geographic analyses}

Using ArcGIS 9.3.1 software (Esri, Redlands, CA, USA), cluster locations with associated nutritional indicator data were analysed. Spatial analyses were conducted to determine the level of clustering of BMI and BAP values. In order to determine if high or low BMI and BAP values were significantly clustered for mothers and children in
Kenya (research question \#1), the global clustering statistic termed the Getis-Ord General G was employed. This statistic determines if there is a significant clustering of high or low values of a particular variable based on a measure of distance between observations or a neighbourhood association among those observations. The variable values of concern in the present research are the BMI values for mothers and the BAP values for children. As shown in equation (1), this statistic is a ratio where the numerator is comprised of the sum of the multiplied variable values of all observations, further multiplied by a weight representing the distance between the observations. The denominator consists of the sum of the multiplied variable values of all observations without regard to the distance between them:

$$
G(d)=\frac{\sum_{i=1}^{N} \sum_{j=1 ; j \neq i}^{N} w_{i j} x_{i} x_{j}}{\sum_{i=1}^{N} \sum_{j=1 ; j \neq i}^{N} x_{i} x_{j}}
$$

where $G(d)$ is the Getis-Ord General G value at critical distance (or neighbourhood) $d ; N$ is the number of geographical areas; $i$ and $j$ are indices of geographical areas; $x_{i}$ is the variable value at area $i ; x_{j}$ is the variable value at area $j$; and $w_{i j}$ is the association weight for areas $i$ and $j$ at distance $d$.

It is imperative that a weighting method is chosen that is appropriate to the spatial nature of the observations. For the research at hand the weights can be defined through the use of a fixed distance neighbourhood method. This method assigns a binary $(0,1)$ weight value to each pair of observations based on whether or not the observations are within the critical neighbourhood distance. This method demands the assumption that observations taken at the same data collection location be considered neighbours. This is intuitive, however, since those residing near the collection location are most likely to actually be neighbours.

For the present research a range of neighbourhood distances were tested for two reasons:

1. There is no known value of what constitutes a neighbourhood in Kenya in the literature, and there are no secondary data from which to derive neighbourhoods (e.g. parcel data, voting data).

2. If a single neighbourhood value was determined, it would almost certainly not apply to all areas of Kenya and therefore would not be appropriate for the current national-scale analysis.

Therefore, in the present research the $G(d)$ statistic was applied across a range of distance values in order to determine if the clustering (if any) is robust across a range of potential neighbourhood definitions.

It is insufficient to simply test for the presence of clustering without an accompanying test of the significance of that clustering. To do so, a null hypothesis of 
complete spatial randomness is created. That is, the assumption is that any clustering that is present in the BMI or BAP values is the result of random chance. This assumption was tested by examining the deviation from the expected value of the $\mathrm{G}(d)$ statistic using equation (2):

$$
E[G(d)]=\frac{\sum_{i=1}^{N} \sum_{j=1 ; j \neq i}^{N} w_{i j}}{N(N-1)}
$$

This deviation was converted to a $Z$-score where a positive $Z$-score indicates clustering of high variable values (BMI or BAP values in the case of the present research) and a negative $Z$-score indicates clustering of low variable values. A $Z$-score near zero indicates no apparent clustering within the study area. The higher (or lower) the $Z$-score, the stronger the intensity of the clustering ${ }^{(21)}$.

In order to address the issue of the geographic distribution of mothers and children who comprise clusters of high or low BMI or BAP values (research question \#2), a local version of the Getis-Ord statistic, the Gi* Statistic, was employed. This statistic generates a value for each observation that represents the extent to which that observation is clustered with other observations that have similar variable values (either high or low). A significance test analogous to the one described above in the global case was applied to each observation. The advantage of the local test is that the individual observations can be mapped, with the $Z$-score values employed as the map theme. This allows visual inspection of the clustering, and interpretation of the results with regard to other spatial distributions. For example, this allows an examination of the clustering of overweight mothers with underweight children in the same regions of the country (research question \#3). Moreover this allows us to compare the spatial distributions with the locations of other natural and man-made features (e.g. the location of cities, borders, natural features).

\section{Results}

We begin with an analysis of the 2003 DHS data and compare these results with those generated from the 2009 data at the end of this section. With regard to research question \#1, which considers the level of clustering of BMI and BAP values among mothers and children, there is extremely strong evidence that high values show strong clustering. Figure 2 shows the $Z$-score values for the Getis-Ord General G statistic for both mothers and children over the range of tested neighbourhood distances (1000 m to $120000 \mathrm{~m}$ ). At every distance tested, clustering of high BAP values for the children was found to be significant at the $0 \cdot 01$ level. Similar results were found for the mothers' BMI values, with clustering of high BMI values found at nearly all distances. However, that clustering

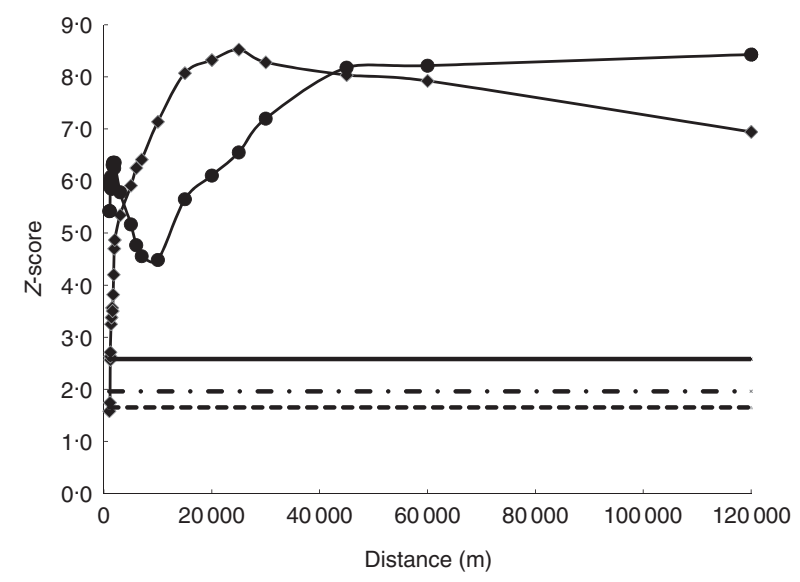

Fig. 2 Getis-Ord Z-scores showing clustering of high BMI and BMI-for-age percentile values among mother-child pairs ( $n$ 1541), data from the Kenya 2003 Demographic and Health Survey (- - Z-score of mothers; - - Z- Z-score of children; ---, 0.10 significance level; - - - - 0.05 significance level; $\longrightarrow, 0.01$ significance level)

was not uniformly significant; specifically, the clustering was significant at the $0 \cdot 01$ level for distances from $1185 \mathrm{~m}$ to $120000 \mathrm{~m}$, it was significant at the 0.05 level at the neighbourhood distance of $1100 \mathrm{~m}$, and it was not significant at the distance of $1000 \mathrm{~m}$ at the $0 \cdot 1$ level.

These results may be interpreted to mean that mothers with high BMI values tend to be located near to one another spatially, and children with high BAP values are likewise likely to be spatially clustered. This global statistic that considers observations from the entire country of Kenya did not detect any clustering of low BMI or BAP values at any distance. Therefore, there is no evidence that underweight mothers or underweight children are spatially clustered.

Regarding research questions \#2 and \#3, Figs 3 and 4 clearly demonstrate the spatial patterns and co-location tendencies of the different populations under consideration. These figures show the local Getis-Ord Gi* values for the individual cluster locations for the 2003 data. These values have been grouped based on the significance values of the observations. Any $Z$-score that is significant at the $0 \cdot 1$ level is included as demonstrating significant clustering. Since there are no previous studies employing the Getis-Ord statistic for the investigation of BMI or BAP values this cut-off value was chosen based on its common use in other research areas. Further research is needed to determine an appropriate standard value for analyses of this type. However, there are clear groupings of high BMI values for mothers in Mombasa. There is very little evidence of clustering of overweight mothers in the Kisumu region. Conversely, there is strong evidence of clustering of overweight children in the Kisumu region while there is none in Mombasa. In Nairobi it appears that there is clustering of both overweight mothers and overweight children. Small groupings of low BMI values 


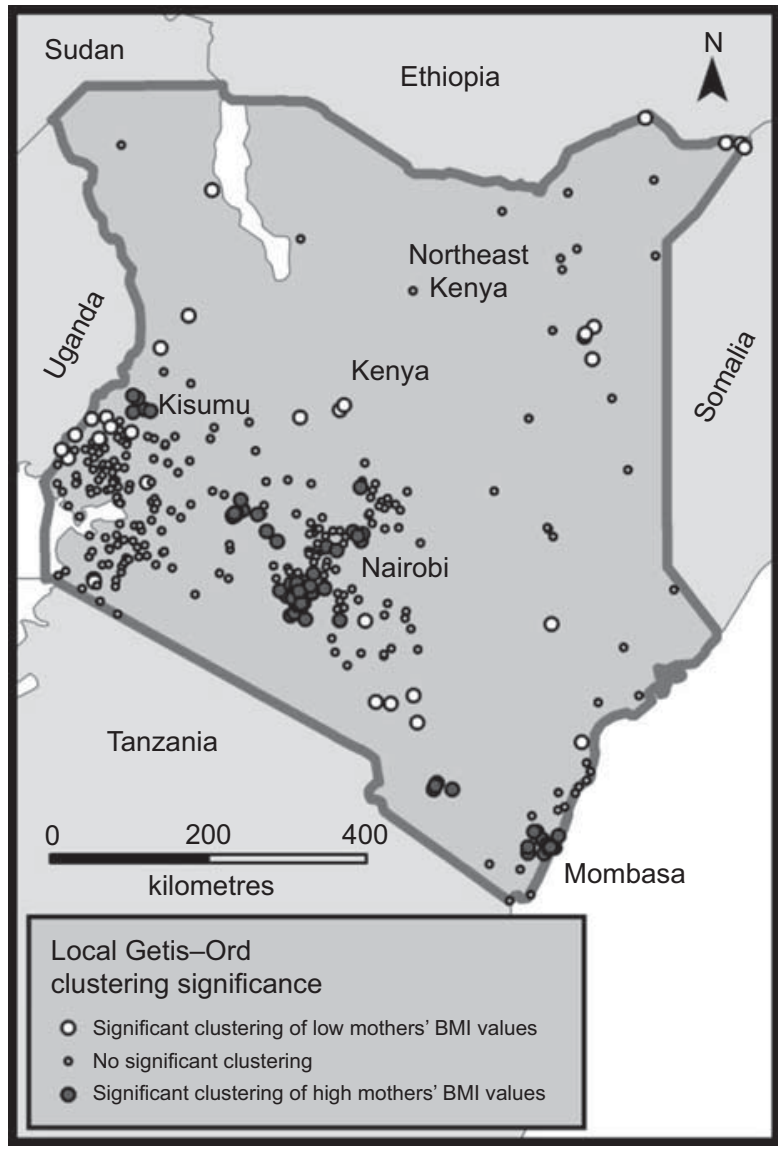

Fig. 3 Local Getis-Ord Gi* values for clustering of mothers' $\mathrm{BMI}(n$ 1541), data from the Kenya 2003 Demographic and Health Survey

for mothers and low BAP values for children are scattered across the rural areas of Kenya. Of particular note is that the observations taken immediately along the borders of Ethiopia, Somalia and Uganda (believed to be formal or informal refugee camps/settlements) show significant clustering of low BMI values among mothers, while underweight children appear to be clustered in more central rural areas. Although Figs 3 and 4 only show the spatial pattern for a neighbourhood distance of $30 \mathrm{~km}$, the same range of distances shown in Fig. 2 were tested with very similar results at each distance.

This spatial analysis was repeated using the 2009 DHS data to determine if clustering of the nutritional indicators persisted through time. It was found that the results were nearly identical between the two data sets. More specifically, in the case of mothers, high BMI values were observed clustering in both Mombasa and Nairobi, and there were still clusters of low BMI values in the Kisumu region. We saw some persistence of clusters of low BMI values in rural areas although fewer were directly on the borders. In the case of children, there was again no evidence of significant clustering in Mombasa in 2009. Clusters of high BAP values were again observed in

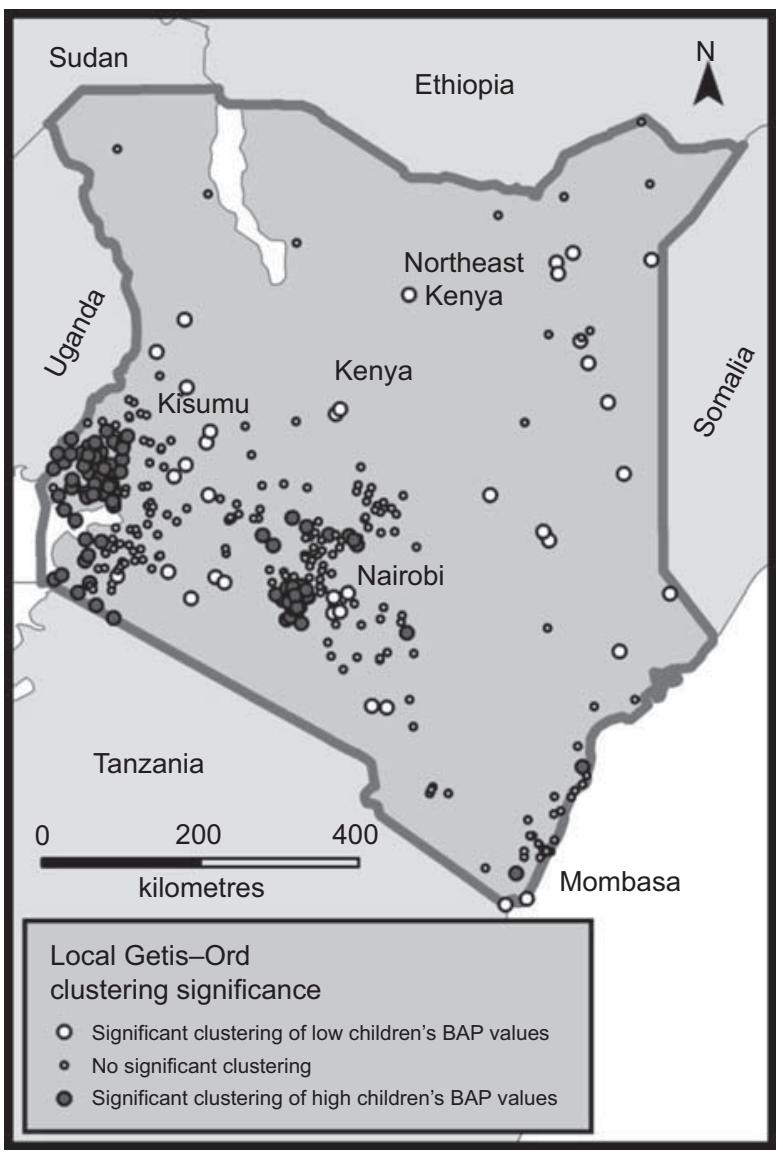

Fig. 4 Local Getis-Ord Gi* values for clustering of children's $\mathrm{BMI}$-for-age percentile values ( $n$ 1541), data from the Kenya 2003 Demographic and Health Survey

Table 1 Nine classifications for mothers' BMI and children's BMIfor-age percentile values ( $n$ 1541), data from the Kenya 2003 Demographic and Health Survey

\begin{tabular}{lccc}
\hline & $\begin{array}{c}\text { Child } \\
\text { underweight } \\
(\%)\end{array}$ & $\begin{array}{c}\text { Child } \\
\text { normal- } \\
\text { weight (\%) }\end{array}$ & $\begin{array}{c}\text { Child } \\
\text { overweight } \\
(\%)\end{array}$ \\
\hline Mother underweight & $2 \cdot 0$ & $10 \cdot 7$ & 1.3 \\
Mother normal-weight & 4.9 & 49.3 & $10 \cdot 7$ \\
Mother overweight & 0.6 & 14.8 & 5.7 \\
\hline
\end{tabular}

Nairobi and the central Kisumu region. Similarly, clusters of low BAP values were observed in the eastern/rural Kisumu area and throughout the rural areas of the country.

Finally, Table 1 presents a frequency table concerning BMI and BAP status of both mothers and children. With regard to the relationship of the mothers' BMI to the children's BAP in the entire country, the categories in order of prevalence are: (i) normal-weight mothers and normal-weight children; (ii) overweight mothers with normal-weight children; (iii) normal weight mothers and underweight children; and (iv) normal-weight mothers and overweight children. 


\section{Discussion}

Obesity has increasingly become an epidemic in industrialized nations, particularly in the USA, where one out of every three adults is obese. However, the USA is not alone with this emerging public health crisis. Much of the blame in the developed world is put on increased television watching, larger portion sizes and the proliferation of cheap fast-food restaurants, all contributing to a toxic food environment ${ }^{(2)}$. It has been found that fast food in the form of street food has become an important source of energy for urban Kenyan populations ${ }^{(22)}$. Furthermore, in the past 15 years a growing trend of obesity has been noted in developing and transitional countries, and most particularly in Latin America ${ }^{(23)}$. However, little research has explored the prevalence of overweight and obesity in sub-Saharan Africa. The findings reported herein are the first to geographically examine indicators of nutritional status among Kenyan mothers and children.

The present research adds to the literature by revealing the clear geographic patterns underlying this phenomenon. When examining the entire country, our results show that mothers and children with high BMI or BAP values live in the same communities and mothers and children with low BMI or BAP values are more dispersed around the country. This global result is confirmed by local spatial analyses demonstrating that overweight/ obese mothers and children tend to cluster more in urban areas including near Nairobi, while underweight mothers and children appear to cluster in the Northeast area and in more rural regions. Further, overweight mothers cluster on the east coast near Mombasa, while there is no evidence of clusters of non-normal-weight children in that region. These results were consistent over time when both 2003 and 2009 DHS data were examined. While rural clusters of low BMI values persisted in results from the 2009 data set, the absence of such clusters directly on Kenya's international borders suggests better conditions in refugee camps as compared with 2003. Moreover, food insecurity was more widespread in 2009 with rains having failed in much of the country ${ }^{(24)}$. This could explain the presence of clusters of low BMI values in areas not necessarily dominated by the presence of refugees. Given the current situation with famine on the Kenyan-Somali border this situation may change once again, giving even further significance to this work in terms of potential policy implications.

These findings confirm the results reported by Gewa ${ }^{(13)}$ and others ${ }^{(25,26)}$ that showed a greater prevalence of overweight and obese mothers and children living in urban areas, even when controlling for socio-economic factors $^{(10,27)}$. Thus, in Africa, there may be more specific factors related to urbanization which contribute to greater food intake and more sedentarism, and thus greater obesity. Since the findings from Gewa's earlier study did not reveal wealth or socio-economic status as a determinant for overweight or obesity in children in this population, it is clear that it is imperative to better understand the influence of urbanization on the growth of overweight and obesity in these transitional populations in Kenya.

It is possible that there are cultural differences within countries such as Kenya that can explain the variation in clusters of high (or low) BMI and BAP values. For example, there is some evidence that religious ${ }^{(28)}$ and cultural $^{(29,30)}$ values can influence obesity rates ${ }^{(31)}$. Differences in such values across regions in Kenya may influence communities' perception of health and acceptable body images among women and children. This is pertinent to research question \#3 since there is a pattern of overweight mothers being clustered in the Mombasa region (which is culturally distinct from the rest of Kenya) while there is no evidence of underweight children clustering in that same region. Conversely, in the Kisumu region there is evidence of clustering of overweight children coupled with no evidence of clustering of overweight mothers. The frequency table shows us that this relationship does exist although it is relatively infrequent $(0 \cdot 6 \%)$. However, what does appear in greater frequency is the number of overweight/obese mothers and children of normal weight.

These findings clearly demonstrate the influence of community/neighbourhood geographic factors on nutritional status. While not previously explored in Kenya, such relationships are not uncommon in high-income countries. In the USA, relatively recent research has explored the influence of neighbourhood characteristics on the rise of obesity among children. While still inconclusive, many of these studies suggest that neighbourhood characteristics including safety issues, access to healthy food stores and restaurants, and access to parks and recreation facilities may influence the prevalence of childhood obesity ${ }^{(32,33)}$. However, no such studies have been conducted in Kenya or other regions of Africa, as the transition to overweight and obesity is a fairly recent occurrence and complicated by the dual problem of stunting and wasting which remains critical throughout the country. Further studies link the social networks of individuals to overweight and obesity, such that there are similar patterns of nutritional status within social and family networks ${ }^{(34)}$.

The findings showing a greater prevalence of underweight in the Northeast region of Kenya are quite consistent with studies showing greater risk of undernutrition among children in rural remote regions of Africa. Further, not only do the desert climate and high poverty of this region contribute to poor nutritional status, the Northeast region of Kenya has been a major area of conflict and unrest particularly along the Kenya-Somali border. This area has also been overwhelmed with refugees and internally displaced persons. For example, as recently as 2005 armed conflict between the two major clans in the region (Garre and Marehan) exploded along the 
Kenya-Somali border over the disputed town of El Wak, Somalia. The fighting produced an estimated 17000 refugees fleeing into Kenya ${ }^{(35)}$. Thus the high prevalence of undernutrition among both mothers and children in this region is not surprising.

\section{Conclusions}

While Gewa ${ }^{(13)}$ has examined the nutritional and sociodemographic determinants of overweight and obesity among children in Kenya, the present study is the first to examine the geographic distribution of the growing problem of overweight/obesity and the continuing struggle with undernutrition in Kenya. The findings show there is geographic variability as well as some defined patterns concerning the distribution of nutritional indicators among mothers and children in Kenya, and suggest the need for further geographic analyses concerning the potential factors which influence nutrition in this population. Moreover the research demonstrates that clustering over time can be investigated and the spatial components of the nutrition transition can thus be documented. In addition, the methods used in the present research may be easily applied to other DHS survey data in order to begin to understand geographic determinants of health in low-income countries.

\section{Acknowledgements}

This work was partially supported by the National Research University Project of Thailand Office of Higher Education Commission. The authors know of no conflict of interest. All authors contributed to the writing and analysis presented here. The extent of author contributions is reflected in the order of authorship. The authors would like to acknowledge several faculty members and students in Geography and Geoinformation Science at George Mason University for their input and advice on the preparation of the manuscript.

\section{References}

1. World Health Organization (2011) Obesity and Overweight, Fact Sheet Number 311. http://www.who.int/mediacentre/ factsheets/fs311/en/ (accessed January 2012).

2. Popkin BM \& Gordon-Larsen P (2004) The nutrition transition: worldwide obesity dynamics and their determinants. Int J Obes Relat Metab Disord 28, Suppl. 3, S2-S9.

3. World Health Organization (2012) Facts and Figures. http:// www.euro.who.int/en/what-we-do/health-topics/diseaseprevention/nutrition/facts-and-figures (accessed January 2012).

4. Popkin BM (2001) The nutrition transition and obesity in the developing world. J Nutr 131, issue 3, 871S-873S.

5. Pieters M \& Vorster HH (2008) Nutrition and hemostasis: a focus on urbanization in South Africa. Mol Nutr Food Res 52, 164-172.
6. Letamo $G$ (2011) The prevalence of, and factors associated with, overweight and obesity in Botswana. J Biosoc Sci $\mathbf{4 3}$, 75-84.

7. Pasquet P, Temgoua LS, Melaman-Sego F et al. (2003) Prevalence of overweight and obesity for urban adults in Cameroon. Ann Hum Biol 30, 551-562.

8. Ntandou G, Delisle H, Agueh V et al. (2009) Abdominal obesity explains the positive rural-urban gradient in the prevalence of the metabolic syndrome in Benin, West Africa. Nutr Res 29, 180-189.

9. Kimani-Murage EW, Kahn K, Pettifor JM et al. (2010) The prevalence of stunting, overweight and obesity, and metabolic disease risk in rural South African children. BMC Public Health 10, 158.

10. Bouzitou G, Fayomi B \& Delisle H (2005) Child malnutrition and maternal overweight in same households in poor urban areas of Benin. Sante 15, 263-270.

11. Garrett J \& Ruel MT (2005) The coexistence of child undernutrition and maternal overweight: prevalence, hypotheses, and programme and policy implications. Matern Child Nutr 1, 185-196.

12. Kitsantas P, Pawloski LR \& Gaffney KF (2010) Maternal prepregnancy body mass index in relation to Hispanic preschooler overweight/obesity. Eur J Pediatr 169, 1361-1368.

13. Gewa CA (2010) Childhood overweight and obesity among Kenyan pre-school children: association with maternal and early child nutritional factors. Public Health Nutr 13, 496-503.

14. Dietz PM, Callaghan WM, Cogswell ME et al. (2006) Combined effects of prepregnancy body mass index and weight gain during pregnancy on the risk of preterm delivery. Epidemiology 17, 170-177.

15. Whitaker RC (2004) Predicting preschooler obesity at birth: the role of maternal obesity in early pregnancy. Pediatrics 114, e29-e36.

16. Wrotniak BH, Shults J, Butts S et al. (2008) Gestational weight gain and risk of overweight in the offspring at age $7 \mathrm{y}$ in a multicenter, multiethnic cohort study. Am J Clin Nutr 87, 1818-1824.

17. Holmes J, de Pineres S \& Curtin K (2006) Drugs, violence, and development in Colombia: a department-level analysis. Lat Am Polit Soc 48, 157-184.

18. Hill RL \& Curtin KM (2011) Solar powered light emitting diode distribution in developing countries: an assessment of potential distribution sites in rural Cambodia using network analyses. Soc Econ Plann Sci 45, 48-57.

19. de Onis M (2003) The WHO Multicentre Growth Reference Study (MGRS). http://www.who.int/childgrowth/mgrs/en/ (accessed December 2010).

20. World Health Organization (2006) BMI classification. http://apps.who.int/bmi/index.jsp?introPage =intro_3.html (accessed January 2012).

21. Getis A \& Ord JK (1992) The analysis of spatial association by use of distance statistics. Geogr Anal 24, 189-206.

22. van't Reit H, den Hartog AP \& van Staveren WA (2002) Nonhome prepared foods: contribution to energy and nutrient intake of consumers living in two low-income areas in Nairobi. Public Health Nutr 5, 515-522.

23. Pan American Health Organization (2000) Obesity and Poverty: A New Public Health Challenge. Washington, DC: Pan American Health Organization.

24. Gettleman J (2009) Lush Land Dries Up, Withering Kenya's Hopes. The New York Times, 7 September; available at http:// www.nytimes.com/2009/09/08/world/africa/08kenya.html

25. Ziraba AK, Fotso JC \& Ochako R (2009) Overweight and obesity in urban Africa: a problem of the rich or the poor? BMC Public Health 9, 465.

26. Christensen DL, Eis J, Hansen AW et al. (2008) Obesity and regional fat distribution in Kenyan populations: impact of ethnicity and urbanization. Ann Hum Biol 35, 232-249. 
27. Pawloski LR \& Kitsantas P (2008) Classification tree analysis of stunting in Malian adolescent girls. Am J Hum Biol 20, 285-291.

28. Fraser D, Bilenko N, Vardy H et al. (2008) Differences in food intake and disparity in obesity rates between adult Jews and Bedouins in southern Israel. Ethn Dis 18, 13-18.

29. Harter P (2004) Mauritania's 'wife-fattening' farm. $B B C$ News, 26 January; available at http://news.bbc.co.uk/2/hi/ 3429903.stm

30. Brink P (1989) The fattening room among the Annang of Nigeria. Med Anthopol 12, 131-143.

31. Caprio S, Daniels SR, Drewnowski A et al. (2008) Influence of race, ethnicity, and culture on childhood obesity: implications for prevention and treatment. Diabetes Care 31, 2211-2221.
32. Franco M, Diezroux A, Glass T et al. (2008) Neighborhood characteristics and availability of healthy foods in Baltimore. Am I Prev Med 35, 561-567.

33. Singh GK, Siahpush M \& Kogan MD (2010) Neighborhood socioeconomic conditions, built environments, and childhood obesity. Health Aff (Millwood) 29, 503-512.

34. Christakis N \& Fowler J (2007) The spread of obesity in a large social network over 32 years. N Engl J Med 357, $370-379$

35. United States Agency for International Development (2005) Kenya-Somali Conflict Border Analysis. http:// www.somali-jna.org/downloads/Kenya-Somalia\%20Menkhaus \%20\%282\%29.pdf (accessed January 2012). 